\title{
Discursos SOBRE REgUlaÇÃo E GOVERNANÇA ALGORÍTMICA
}

\author{
Sergio Amadeu da SILVEIRA*
}

\begin{abstract}
RESUMO: O texto traz as práticas discursivas das principais pesquisas sobre a regulação e a governança dos algoritmos. Discorre sobre os elementos fundamentais que articulam os discursos em torno de sistemas algorítmicos e suas expressões como Big Data, machine learning e Inteligência Artificial (IA). Identifica os principais enunciados e expõe as controvérsias sobre regulação e as contraposições discursivas sobre os riscos e benefícios da implementação generalizada dos sistemas algorítmicos.
\end{abstract}

PALAVRAS-CHAVE: Sistemas algorítmicos. Governança algorítmica. Regulação algorítmica. Algoritmos. Inteligência Artificial.

\section{Introdução}

É preciso diferenciar a regulação feita pelos algoritmos da realizada sobre os algoritmos. A primeira é a agência dos algoritmos sobre pessoas e coisas e a segunda trata da ação das pessoas via dispositivos normativos e legais sobre os algoritmos. Enquanto uma trata de como alguns grupos compreendem e controlam processos e situações que envolvem demais pessoas e coisas por intermédio dos algoritmos, a outra tem como objetivo controlar socialmente as ações exercidas pelos algoritmos.

Os algoritmos são objeto da perspectiva regulatória dada a dimensão que alcançaram na sociedade. A expansão da sociedade informacional está diretamente relacionada à expansão de máquinas computacionais e softwares. Estes últimos são

UFABC - Universidade Federal do ABC. São Bernardo do Campo - SP - Brasil. 09606-045 - sergio. amadeu@ufabc.edu.br. https://orcid.org/0000-0003-1029-9133.

Bolsista de Produtividade em Pesquisa do CNPq - Nível 2. 
formados por algoritmos. O desenvolvimento dos programas para os dispositivos de processamento de informação durante suas fases iniciais foi determinado pelos algoritmos que seguiam regras. A partir da segunda década deste século, se disseminaram programas e sistemas definidos por algoritmos que se alteram em função dos dados e das finalidades que os delimitam. A propagação do uso dos sistemas algoritmos que aprendem com dados propiciou a emergência dos modelos de negócios orientados a dados (data-driven business models), do marketing dirigido por dados (data-driven marketing), governança baseada em dados (data-driven governance), entre outros. Trata-se do culto aos dados que o Big Data propiciou ao atualizar as velhas perspectivas positivistas que podem ser expressas no enunciado "deixar os dados falarem" (MAYER-SCHONBERGER \& CUKIER, 2013, p.6).

Os algoritmos servem à atividade automatizada. Sejam baseados em regras ou em dados, os algoritmos têm vocação para a ação sem a necessidade da intervenção humana. Por isso, suas implicações sociais são grandes e os discursos que engajam e caracterizam os sistemas algoritmos são, em geral: de apoio às atividades humanas como se fossem ferramentas; de ampliação exponencial ou qualitativa das ações efetuadas por humanos (produtividade) e de criação de novas possibilidades e ações impossíveis de serem executadas por pessoas. Os discursos sobre os algoritmos ou sobre os sistemas algorítmicos que compõem o Big Data, o aprendizado de máquina e a Inteligência Artificial (IA) são discursos de promoção de suas capacidades, inteligência, velocidade ou empoderamento. Mesmo os discursos críticos, que reforçam os aspectos negativos, estão envoltos pela ideia de superioridade algorítmica, ou seja, da ação autônoma desses dispositivos lógicos.

\section{Regulação e governança}

Quando o consultor e empresário Tim O'Reilly (2013, p.291) escreveu que "algorithmic regulation is an idea whose time has come", estava falando da necessidade dos governos realizarem suas atividades e controles substituindo pessoas (funcionários públicos) por sistemas algorítmicos. Grande parte do discurso acadêmico ao tratar da regulação algorítmica se refere ao sistema de tomada de decisão e de controle de pessoas e coisas, nas empresas e/ou no Estado:

Algorithmic regulation refers to decision-making systems that regulate a domain of activity in order to manage risk or alter behavior through continual computational generation of knowledge by systematically collecting data (in real time on a continuous basis) emitted directly from numerous dynamic components

\footnotetext{
1 “A regulação algorítmica é uma ideia cujo tempo chegou”. (O'REILLY, 2013, p.291, tradução nossa.).
} 
pertaining to the regulated environment in order to identify and, if necessary, automatically refine (or prompt refinement of) the system's operations to attain a pre-specified goal ${ }^{2}$ (YEUNG, 2017, p.3).

Baldwin, Scott e Hood (1998) escreveram que a regulação pode comportar três concepções principais, conforme disciplinas acadêmicas diferentes. Nesse sentido, essas concepções podem também ser compreendidas como uma organização de enunciados que compõem práticas discursivas distintas. A primeira concepção ou prática discursiva vê a regulação como a definição de um conjunto de regras autoritativas e mecanismos para a o seu cumprimento. A segunda, como os esforços das agências estatais para definir a direção da economia. A terceira, envolve todos os mecanismos de controle social, inclusive, os não-intencionais e não estatais.

O discurso da regulação algorítmica traz a questão da fixação de padrões de conduta e ao monitoramento e a coleta de dados para se efetivar em sistemas mais sofisticados tais como o de machine learning (ML). A regulação pelos algoritmos pode levantar novas composições discursivas que carregam controvérsias sobre a discricionariedade, flexibilidade e performatividade dos sistemas algorítmicos, como será tratado mais à frente.

Uma das questões controversas é a que define como inócua a tentativa de regular as operações algorítmicas dirigidas por dados, como no caso do aprendizado profundo, das redes neurais e em certos modelos de IA. O discurso da impossibilidade de explicação dos procedimentos realizados pelo sistema algorítmico para se atingir um resultado levaria a impossibilidade de seu controle, pois ele age segundo a estrutura de dados que obtém.

A pesquisadora Karen Yeung faz a aproximação de regulação e governança a partir da perspectiva de Julia Black (2014) que define regulação como governança regulatória. Assim, a regulação algorítmica para Yeung $(2017$, p.3) seria a "intentional attempts to manage risk or alter behavior in order to achieve some pre-specified goal"3. Em primeiro lugar, Yeung (2017) define que a regulamentação não deve ser considerada como uma atividade exclusivamente estatal. O Facebook também regula o comportamento de seus usuários. Em segundo lugar, que existe a regulação pelo direcionamento do comportamento de um determinado serviço. Isso ocorre com o Uber que oferece vantagens e incentivos para que os motoristas se

\footnotetext{
2 A regulação algorítmica refere-se a sistemas de tomada de decisão que regulam um domínio de atividade para gerenciar riscos ou alterar o comportamento por meio da geração computacional contínua de conhecimento, coletando sistematicamente dados (em tempo real e continuamente) emitidos diretamente de vários componentes dinâmicos pertencentes ao ambiente regulado para identificar e, se necessário, refinar automaticamente (ou refinar rapidamente) as operações do sistema para atingir uma meta pré-especificada. (YEUNG, 2017, p.3, tradução nossa)

3 "tentativas intencionais de gerenciar riscos ou alterar comportamentos, a fim de alcançar algum objetivo pré-especificado". (YEUNG, 2017, p.3, tradução nossa).
} 
dirijam a determinados bairros. Em terceiro, considera que a regulamentação é uma atividade intencional. Não há como atingir uma meta regulatória sem um dirigente do sistema regulador, ou seja, ele é quem determina as metas do sistema regulatório.

\section{A regulação não-estatal}

O discurso da regulação não-estatal é compatível com um ambiente hegemonicamente neoliberal (DARDOT; LAVAL, 2017). Existem discursos, principalmente no segmento corporativo e nas consultorias sobre a inadequação de uma regulação forte, entendida como regulamentação ou aprovação de leis que afetem o desenvolvimento tecnológico (THE WHITE HOUSE OFFICE, 2018). Mais recentemente, surgiu também outro discurso que clama pela corregulação, forma de regulação híbrida, uma vez que reconhece os problemas da regulação estatal sobre os sistemas algorítmicos, mas que também identifica que "we have little reason to believe the companies we depend on will act in our interest in the absence of regulatory oversight"4 (SANDVIG et al., 2014, p.7). Christopher T. Marsden (2012) afirma que a corregulação se dá pela articulação das múltiplas partes interessadas no processo, objeto ou atividade a ser regulado, o que dá maior legitimidade às definições. "The state, and stakeholder groups including consumers, are stated to explicitly form part of the institutional setting for regulation"5 (MARSDEN, 2012, p.211). A corregulação tem ganhado força entre ativistas digitais e instituições como o Centre for European Policy Studies, organização independente sediada em Bruxelas.

Os discursos de regulação estatal ou hibrida, seja de corregulação, seja de regulação realizada por organizações independentes do Estado, seja auto regulação fiscalizada por auditorias e garantida por termos de compromisso e de conduta (compliance) não destacam que ao se tornarem atividades fundamentais e permanentes criam um mercado de regulação. As exigências da regulação e a atividade reguladora podem fomentar a criação de mercados de regulação: consultores, auditores, especialistas em especificações, em produção de medidas e documentos, entre outras. Um exemplo é o que está ocorrendo com a aplicação da Lei Geral de Proteção de Dados, sancionada no Brasil em 2018. Todas as empresas e instituições que armazenam e/ou manipulam dados pessoais terão que se adequar aos seus termos.

\footnotetext{
4 "temos poucas razões para acreditar que as empresas das quais dependemos agirão em nosso interesse na ausência de supervisão regulatória". (SANDVIG et al., 2014, p.7, tradução nossa).

5 "Afirma-se que o estado e os grupos interessados, incluindo os consumidores, fazem explicitamente parte do cenário institucional de regulamentação". (MARSDEN, 2012, p.211, tradução nossa).
} 
Uma pesquisa no mecanismo de busca do Google da expressão "especializado na Lei Geral de Proteção de dados" obteve 14.700.000 resultados. Em seguida, uma busca da frase "especializado na legislação ambiental" alcançou 7.840.000 resultados. A lei de proteção de dados é recente e gerará uma série de exigências o que explica este grande interesse por ela. A criação de novos negócios a partir da regulação é mais intensa no meio jurídico, como podemos observar no artigo de um dos inúmeros blogs relacionados ao Direito:

Não é apenas o direito das startups que oferece oportunidades de trabalho para o advogado. Olhando pela ótica da pessoa jurídica, toda e qualquer empresa deverá se adequar à Lei Geral de Proteção de Dados Pessoais. E não serão apenas os grandes escritórios de advocacia que prestarão esse serviço às corporações.

Se você é advogado autônomo, ou atua em uma pequena banca, olhe para a sua região, veja quais são os empreendimentos que circundam o seu escritório. Eles podem guardar excelentes oportunidades para você atuar e oferecer um serviço de adequação à LGPD voltado para a realidade deles. (COELHO, 2019, n.p.).

A atividade de regulação é necessariamente restritiva e delimitadora. $\mathrm{O}$ maior grau de tecnicidade do objeto e do processo a ser regulado torna a atividade de regulação mais carente de especialistas e, portanto, pode ter um elevado custo econômico. A regulação dos sistemas algorítmicos até o momento não foi realizada, exceto se a revisão humana de decisões algorítmicas presentes na Lei Geral de Proteção de Dados da União Europeia for considerada a primeira ação regulatória sobre algoritmos.

Não somente devido à dimensão e à quantidade de acesso, mas também pelo modelo de negócios centrado na coleta de dados e na formação de perfis de interesse e consumo, a ação e o gerenciamento das plataformas de relacionamento online, tais como: Facebook, Youtube, Instagram, LinkedIn, entre outras, são realizados por sistemas algorítmicos. Em geral, são utilizados algoritmos de aprendizado de máquina, de linguagem natural, de reconhecimento de imagens, incluindo outros que distribuem mensagens e anúncios.

\section{Perspectivas liberais, deleuzeanas e institucionalistas}

Influenciado por Simondon, Gilles Deleuze considerou que nossas sociedades, nomeadas por ele como de controle, seriam moduladas. Sem uma definição inequívoca, a noção de modulação permite enquadrar os fenômenos de condução do olhar e do visível nas chamadas redes sociais digitais (SILVEIRA, 2019). O Facebook modula os comportamentos distribuindo os conteúdos conforme os perfis 
definidos e armazenados pelos seus sistemas algorítmicos. Mas a modulação se aproxima muito da ideia de controle ou de regulação pelo design ou pela arquitetura da informação.

Inspirada no texto de Richard H. Thaler e Cass R. Sunstein (2009), Nudge: Improving Decisions About Health, Wealth, and Happiness, Karen Yeung (2017) considera que, a partir do chamado Big Data e sua relação com os algoritmos, estão abertas as possibilidades de moldar o contexto da escolha das pessoas. Ao canalizar as atenções, o algoritmo atua oferecendo caminhos específicos, direções a se tomar, como um nudge (em português, cutucar, empurrar, incentivar), uma suave indicação e uma alteração de comportamento de maneira previsível, sem proibições, sendo uma forma suave de controle por design, tal como o ordenamento dos resultados do mecanismo de busca que oferece nos primeiros resultados os links que tendem a ser mais escolhidos, pela posição que ocupam.

The algorithmic analysis of data patterns dynamically configures the targeted individual's choice environment in highly personalised ways, affecting individual users' behaviour and perceptions by subtly moulding the networked user's understanding of the surrounding world. Their distinctly manipulative, if not straightforwardly deceptive, qualities arise from deliberately exploiting systematic cognitive weaknesses which pervade human decision-making to channel behaviour in directions preferred by the choice architect. ${ }^{6}$ (YEUNG, 2017, p.130).

O discurso acadêmico de orientação liberal tem trazido a crítica ao que considera um exagero dos pesquisadores de influência foucaultiana-deleuzeana e dos teóricos dos estudos de vigilância. $\mathrm{O}$ foco do ataque liberal e neoliberal está na minimização dos riscos que os sistemas de escolha e de decisão algorítmicos possam gerar. Eric Goldman (2006) considera, por exemplo, que a personalização e o viés dos mecanismos de pesquisa podem ser uma consequência benéfica, uma vez que otimiza o conteúdo para seus usuários. Além disso, a "regulatory intervention could counterproductively degrade search engines' value to searchers. Whatever the adverse consequences of search engine bias, the consequences of regulatory correction are probably worse." (GOLDMAN, 2006, p.198).

\footnotetext{
6 A análise algorítmica dos padrões de dados configura dinamicamente o ambiente de escolha do indivíduo alvo de maneiras altamente personalizadas, afetando o comportamento e as percepções dos usuários individuais, moldando sutilmente a compreensão do usuário em rede do mundo circundante. Suas qualidades distintamente manipuladoras, se não objetivamente enganosas, surgem da exploração deliberada de fraquezas cognitivas sistemáticas que permeiam a tomada de decisões humanas para canalizar o comportamento nas direções preferidas pelo arquiteto escolhido. (YEUNG, 2017, p.130, tradução nossa).

7 "a intervenção regulatória pode degradar de forma contraproducente o valor dos mecanismos de pesquisa para os pesquisadores. Quaisquer que sejam as consequências adversas do viés do
} 
A rejeição aos controles estatais e à regulação mais forte, muito comum, por exemplo, no debate acadêmico sobre a governança da Internet, é um discurso presente não somente a partir do pensamento liberal e das corporações, envolve também a perspectiva mais autonomista e participativa. Adiante serão apresentados enunciados que começam a se entrelaçar e a se constituir em uma prática discursiva que considera a necessidade regulatória, mas reivindica a participação dos regulados e dos segmentos da sociedade interessados.

Frank Pasquale (2015) trabalhou em seu núcleo discursivo a questão da opacidade ou invisibilidade dos sistemas algorítmicos no texto The Black Box Society e das técnicas de ofuscação realizadas pelas instituições que utilizam esses algoritmos, o que remete diretamente à ausência de transparência e de accountability (em português, responsabilidade). Esses dois elementos discursivos têm sido tratados por diversos autores e organizações, fazendo parte de uma longa tradição de debates sobre o Estado (BOBBIO, 1997). Em relação aos sistemas algoritmos da chamada Inteligência Artificial, a questão do accountability se tornou um dos pontos de controvérsia, dada a impossibilidade de acompanhar, compreender e explicar o seu funcionamento. Entre os defensores da necessidade de explicação e de abertura para a fiscalização social temos diversos argumentos apresentados pelo pesquisador de mídia, Nicholas Diakopoulos (2014, 2015, 2016).

Em 2015, o Tow Center for Digital Journalism da Universidade de Columbia realizou o workshop sobre a transparência algorítmica na Mídia. Diakopoulos (2016) destacou que 50 especialistas da mídia e da academia discutiram conteúdos de notícias gerados automaticamente, simulações de modelagem narrativa, curadoria algorítmica e diversas dimensões da filtragem e classificação dos algoritmos. No encontro, cinco categorias - envolvimento humano, dados, modelo, inferências e presença algorítmica - foram selecionadas e preocupações foram apresentadas para cada uma delas (DIAKOPOULOS, 2016, p.60-61). É importante ressaltar que o discurso da transparência se vincula diretamente à necessidade de responsabilidade humana e da recusa de que os sistemas algorítmicos não possam ser compreendidos no plano de suas ações, intensões e resultados pretendidos.

At a high level, transparency around human involvement might involve explaining the goal, purpose, and intent of the algorithm, including editorial goals and the human editorial process or social context crucible from which the algorithm was cast. Who at your company has direct control over the algorithm? Who has oversight and is accountable? Ultimately, we want to identify the authors, or the designers, or the team that created and are behind this thing. (...) Involved

mecanismo de busca, as consequências da correção regulatória provavelmente são piores". (GOLDMAN, 2006, p.198, tradução nossa). 
individuals might feel a greater sense of public responsibility and pressure if their names are on the line. ${ }^{8}$ (DIAKOPOULOS, 2016, p.60).

A ideia de que os algoritmos estão constituindo uma esfera pública automatizada (PASQUALE, 2017), ou seja, operada pelos sistemas algorítmicos e o clamor pela transparência, accountabillity e responsabilização implicam em regulação mais forte, legalmente definida, mesmo que operada ou delineada por organizações fora do Estado. Disso resulta na necessidade de definição de quem deve ser o regulador dos sistemas algorítmicos. Até o momento, apesar dos nomes fantasiosos de sistemas e softwares, os discursos não apresentaram nenhum algoritmo ou sistema algorítmico dono de si, seja no sentido jurídico de proprietário de sua patente ou copyright. Assim, a regulação sobre algoritmos, machine learning e outros modelos de Inteligência Artificial recairá sobre empresas e pessoas. "Desconfie dos mecanismos autorreguláveis das plataformas", escreveu Frank Pasquale (2017, p.27) que propugna pela regulação concretizada na lei e nas ações de agentes legalmente instituídos sobre as corporações que desenvolvem e são proprietárias dos algoritmos:

[...] devemos exigir auditorias de dados tratados por sistemas algorítmicos. Aprendizagem por máquina, análises preditivas ou algoritmos podem ser muito complexos para uma pessoa entender, mas os registros de dados não o são. Sendo assim, eles podem, inclusive, ser auditados algoritmicamente. (PASQUALE, 2017, p.23).

O discurso da regulação tem suas definições e problemas conectados ao discurso da governança algorítmica. A governança dos algoritmos pode ser compreendida como a direção, delimitação, controle, enfim, como regulação sobre os sistemas algorítmicos. Regulação no sentido de processos humanos, sociais, intencionalmente conduzidos para dirigir os sistemas algorítmicos. As investigações sobre governança algorítmica ganharam força a partir de 2013, apesar de alguns pesquisadores das chamadas Ciências Humanas e Sociais terem publicado trabalhos que traziam a temática do controle algorítmico na primeira década do século XXI (INTRONA; NISSENBAUM, 2000; BEER, 2009; ANEESH, 2009).

8 Em um nível alto, a transparência em torno do envolvimento humano pode conter a explicação do objetivo, do propósito e da intenção do algoritmo, incluindo objetivos editoriais e o processo editorial humano ou o contexto social a partir do qual o algoritmo foi lançado. Quem na sua empresa tem controle direto sobre o algoritmo? Quem o supervisiona e é por ele responsável? Por fim, queremos identificar os autores, os designers ou a equipe que criou e está por trás deles. [...]. Os indivíduos envolvidos podem sentir um maior senso de responsabilidade e pressão do público se seus nomes estiverem em jogo. (DIAKOPOULOS, 2016, p.60, tradução nossa). 
Algorithm governance can vary from the strict legal and regulatory viewpoints to a purely technical standpoint. Its focus is often on accountability, transparency, and technical assurances. The resource to a certain governance path can be based on factors such as the nature of the algorithm, its context, or risk analysis. Generally, when a governance option is made it aims to reduce problems caused by the algorithm. It should try to preserve its effectiveness and reduce undesirable outcomes. ${ }^{9}$ (DONEDA; ALMEIDA, 2016, p.61).

O fenômeno da governança algorítmica aparece também como uma tendência histórica da mecanização do poder, da modernização da organização jurídico-burocrática que seguiria o ritmo de progresso da indústria (DANAHER et al, 2017, p.2). Apesar de Aneesh (2009) considerar o controle algorítmico sobre os trabalhadores qualitativamente diferente do controle burocrático, ambos são manifestações de um poder descentralizado ou distribuído sobre pessoas. Todavia, são caracterizados por processos distintos em graus de flexibilidade, discricionariedade, compreensão e visibilidade.

\section{Métodos de estudo da governança dos algoritmos}

O pesquisador Henrique Félix de Souza Machado publicou, em 2018, a primeira compilação realizada no Brasil sobre regulação e governança algorítmica. Ao utilizar o Google Scholar acabou analisando 45 textos que apareciam na busca e se relacionavam com o tema. Constatou uma presença maior nas análises realizadas da tradição foucaultiana (WILLIANSON, 2013; ROUVROY, 2014; MCQUILLAN, 2015), da teoria do Ator-Rede (INTRONA, 2013; MUSIANI 2013; CAMPBELL-VERDUYN et al., 2016) e da perspectiva institucionalista e regulatória (WILLIAMSON, 2014; YEUNG, 2016), além do que, reuniu visões da economia, negócios e produção colaborativa e auto governança (O’REILLY, 2013; PITT et al., 2014), entre outras. O recorte e enquadramento são bastante discutíveis, mas Machado (2018) apresentou uma grande contribuição ao debate acadêmico. A perspectiva marxista estava ausente ou sob forte nebulosidade no debate e no discurso científico sobre os sistemas algorítmicos. Em 2019, Nick Dyer-Witheford, Atle Mikkola Kjøsen and James Steinhoff lançam o livro Inhuman Power: Artificial

\footnotetext{
9 A governança do algoritmo pode variar dos estritos pontos de vista legais e regulatórios até um ponto de vista puramente técnico. Muitas vezes, seu foco é responsabilidade, transparência e garantias técnicas. O recurso para um determinado caminho de governança pode ser baseado em fatores como a natureza do algoritmo, seu contexto ou análise de risco. Geralmente, quando uma opção de governança é feita, ela visa reduzir os problemas causados pelo algoritmo. Deve tentar preservar sua eficácia e reduzir resultados indesejáveis. (DONEDA; ALMEIDA, 2016, p.61, tradução nossa).
} 
Intelligence and the Future of Capitalism em que trazem o debate marxista para tentar compreender se o que os sistemas algorítmicos realizam é ou não trabalho.

Um dos trabalhos mais circunstanciado e abrangente sobre a governança algorítmica está registrado no texto Algorithmic governance: developing a research agenda through the power of collective inteligence (DANAHER et al., 2017). Ele resultou de um workshop multidisciplinar realizado na Queen's University Belfast que utilizou o método interativo de gerenciamento assegurando aos participantes que produzissem uma agenda e estrutura de pesquisa sobre a governança algorítmica. Três aspectos da literatura foram destacados: a) as formas e os modos de governança algorítmica; b) os problemas de governanças algorítmica; c) os métodos para o estudo da governança dos algoritmos (DANAHER et al., 2017).

Do que poderia ser visto como um dos núcleos discursivos que diferenciam tipos distintos de algoritmos que incidem em diferentes formas de controle ou governança, o texto de Danaher et al.(2017) destaca o contraste entre o design de algoritmos top-down (em português, de cima para baixo) e bottom-up (em português, de baixo para cima). No primeiro tipo, o programador ou uma equipe definem pormenorizadamente o conjunto de regras do algoritmo ou dos algoritmos. No segundo tipo, o algoritmo recebe uma regra de aprendizado para que possa ser treinado por uma coleção ou por agrupamento de dados para a partir daí desenvolver as próprias regras. Os algoritmos chamados por Danaher et al (2017) de bottom-up vão constituir o chamado machine learning. Sendo mais rigoroso, os termos "de cima para baixo" e "de baixo para cima" não expressam bem como operam os algoritmos. O primeiro deveria ser "definido por regras previamente programadas" e o segundo por "operado por regras extraídas de dados".

Esse segundo tipo de algoritmo gera maior dificuldades para sua governança. Ele está no coração de grande parte do que vem sendo chamado de Inteligência Artificial. Em geral, a transparência de algoritmos do primeiro tipo não assegura o conhecimento sem testes com estruturas de dados, uma vez que podem conter formulações extremamente complexas. Mas nos algoritmos de segundo tipo a transparência e o conhecimento sobre as operações são extremamente dificultadas pelas alterações de padrão e de regras que eles extraem dos dados. Diferentes estruturas de dados podem gerar diferentes regras para se obter determinadas finalidades. Como seria possível falar de governança nessa situação? Baseando-se em Citron e Pasquale (2014) e em Zarsky (2013), Danaher et al. (2017) expõem a necessidade de como obter a governança nos quatro estágios da governança algorítmica que encontro na revisão da literatura: coleta de dados, processamento, utilização e aprendizado. A participação humana em cada estágio passou a fazer parte dos objetivos dos discursos de governança.

É importante realçar que o discurso acadêmico crítico à opacidade algorítmica e à possibilidade de vieses e injustiças está na base da reivindicação da revisão 
humana das decisões automatizadas. A legislação europeia, disposta no Regulamento Geral sobre a Proteção de Dados (GDPR), é a primeira legislação abrangente que tratou de aspectos da governança e/ou da regulação diretamente relacionado aos sistemas algorítmicos, em especial, ao aprendizado de máquina. Para assegurar o direito a não discriminação e o direito à explicação de decisões tomadas por sistemas automatizados, o artigo 22 da CGPR confere às pessoas o direito de não estarem sujeitas a uma decisão baseada apenas no processamento automatizado, incluindo a criação de perfis.

Above all else, the GDPR is a vital acknowledgement that, when algorithms are deployed in society, few if any decisions are purely "technical." Rather, the ethical design of algorithms requires coordination between technical and philosophical resources of the highest calibre. ${ }^{10}$ (GOODMAN; FLAXMAN, 2017, p.56).

Apesar da maioria dos textos acadêmicos girarem em torno da perspectiva crítica, Danaher et al. ressaltam no texto Algorithmic Governance (2017) que os sistemas algorítmicos podem ser rápidos, eficientes, abrangentes e imparciais como apontam os pesquisadores Pedro Domingos (2015) e Viktor Mayer-Schönberger e Kenneth Cukier (2013). Contudo, as questões críticas presentes nos argumentos que compõem as práticas discursivas acadêmicas são majoritárias na área das Ciências Sociais. Na identificação dos problemas da governança algorítmica, Danaher et al. (2017) destacaram o trabalho de Tal Zarsky(2016), The trouble with algorithmic decisions, por trazer uma taxonomia com pretensões de classificar todas as dificuldades, dilemas e perturbações da perspectiva crítica. Zarsky (2016) considerou que os sistemas de tomada de decisão algorítmicos têm duas propriedades principais: são automatizados e opacos. Danaher et al. (2017, p.3) argumentam que Zarsky (2016) retira dessas propriedades as objeções que sistematizou em dois ramos centrais: 1) da eficiência e 2) da justiça.

Quadro 1 - Governing Algorithms: An Analytical Framework

\begin{tabular}{|c|c|c|}
\hline Problems & Efficiency & Fairness \\
\hline Attributes & Automation & Opacity \\
\hline
\end{tabular}

Fonte: Zarsky, 2016, p.120.

\footnotetext{
${ }^{10}$ Acima de tudo, o GDPR é um reconhecimento vital de que, quando os algoritmos são implantados na sociedade, poucas ou nenhuma decisão é puramente "técnica". Em vez disso, o design ético dos algoritmos requer coordenação entre os recursos técnicos e filosóficos do mais alto calibre (GOODMAN; FLAXMAN, 2017, p.56, tradução nossa).
} 
O trabalho realizado por 15 pesquisadores das áreas de Ciência da Computação, Direito, Biblioteconomia, Filosofia, Geografia, Psicologia, Ciência de Dados, Ciência Política e Sistemas de Informação, sistematizado no texto de Danaher et al. (2017, p.7-14) identificou 12 categorias principais de barreiras à governança algorítmica efetiva e legítima. São elas:

- Opacidade dos algoritmos;

- Tecnoutopia (otimismo tecnológico, acriticidade tecnológica);

- Tecnopessimismo (medo e paralisia diante das possibilidades da tecnologia);

- Incerteza tecnológica;

- Capacidade / conhecimento entre tecnólogos;

- Capacidade dos gestores e servidores públicos;

- Capacidade dos operadores do Direito;

- Complexidade jurídica e institucional;

- Desequilíbrio entre interesses públicos e privados;

- Governança eficaz versus direitos individuais;

- Consciência Ética (ou falta dela);

- Privacidade e consentimento informado.

Para esclarecer as expectativas sobre os possíveis impactos dos algoritmos, o instituto de pesquisa independente Pew Research Center e o Imagining the Internet Center da Universidade Elon realizaram uma pesquisa com especialistas em tecnologia, acadêmicos, profissionais de empresas e líderes governamentais sobre a crescente utilização dos algoritmos e suas implicações no futuro próximo. A pesquisa foi divulgada com o nome de Code-Dependent: Pros and Cons of the Algorithm Age (RAINIE; ANDERSON, 2017). O quadro a seguir mostra os temas que emergem como relevantes na pesquisa. Apresenta as preocupações que constituirão os componentes dos discursos sobre a governança dos algoritmos. O quadro busca uma síntese discursiva. Todavia não há consenso sobre os temas organizados pelos responsáveis pela pesquisa. Há grandes controvérsias como os relatos detalhados da pesquisa demonstram. 
Quadro 2 - Sete principais temas sobre a Era do Algoritmo

\begin{tabular}{|c|c|}
\hline $\begin{array}{l}\text { I - INEVITABILIDADE } \\
\text { DOS ALGORITMOS }\end{array}$ & $\begin{array}{l}\text { Tema } 1 \text { - Algoritmos continuarão a se espalhar por toda parte } \\
\text { - Os benefícios serão visíveis e invisíveis podem levar a uma maior } \\
\text { percepção humana do mundo } \\
\text { - As muitas vantagens de algoritmos são acompanhadas de desafios } \\
\text { Tema } 2 \text { - Coisas boas estão por vir } \\
\text { - As abordagens orientadas a dados para a solução de problemas } \\
\text { serão expandidas } \\
\text { - Processos de código serão refinados e aprimorados; questões éticas } \\
\text { estão sendo trabalhadas } \\
\text { - "Algoritmos não precisam ser perfeitos; eles só precisam ser } \\
\text { melhores que as pessoas" } \\
\text { - No futuro, o mundo poderá ser governado por IA benevolente }\end{array}$ \\
\hline II - PREOCUPAÇÕES & $\begin{array}{l}\text { Tema } 3 \text { - Humanidade e julgamento humano são perdidos } \\
\text { quando dados e modelagem preditiva se tornam primordiais } \\
\text { - Programar principalmente na busca de profistas e eficiências é } \\
\text { uma ameaça } \\
\text { - Algoritmos manipulam pessoas e resultados e até "leem nossas } \\
\text { mentes" } \\
\text { - Tudo isso levará a uma sociedade orientada por lógica falha, mas } \\
\text { inescapável } \\
\text { - Alguns temem que as pessoas possam perder capacidades sofisti- } \\
\text { cadas de tomada de decisão e inteligência local. } \\
\text { - À medida que o código assume sistemas complexos, os humanos } \\
\text { ficam de fora do circuito } \\
\text { - As soluções devem incluir o respeito pelo indivíduo } \\
\text { Tema } 4 \text { - Existem vieses em sistemas organizados por algoritmos } \\
\text { - Algoritmos refletem os vieses de programadores e conjuntos de } \\
\text { dados } \\
\text { - Os algoritmos dependem de dados frequentemente limitados, } \\
\text { deficientes ou incorretos } \\
\text { Tema } 5 \text { - Categorizações algorítmicas aprofundam divisões } \\
\text { - Os desfavorecidos provavelmente serão ainda mais prejudicados. } \\
\text { - Algoritmos criam bolhas de filtro e silos moldados por coletores } \\
\text { de dados corporativos. Eles limitam a exposição das pessoas a uma } \\
\text { gama mais ampla de ideias e informações confiáveis e eliminam o } \\
\text { acaso. } \\
\text { Tema } 6 \text { - O desemprego aumentará } \\
\text { - Algoritmos mais inteligentes e eficientes substituirão muitas } \\
\text { atividades de trabalho humano. } \\
\text { - Alguns buscam um sistema econômico global redefinido para } \\
\text { apoiar a humanidade. }\end{array}$ \\
\hline
\end{tabular}




\begin{tabular}{|l|l|}
\hline III - DESAFIOS SOCIAIS & $\begin{array}{l}\text { Tema } 7 \text { - Cresce a necessidade de alfabetização algorítmica, } \\
\text { transparência e supervisão. } \\
\text { - Começa com a alfabetização de algoritmos - isso vai além da } \\
\text { alfabetização digital básica } \\
\text { - As pessoas pedem processos de responsabilização, supervisão e } \\
\text { transparência } \\
\text { - Muitos são pessimistas quanto às perspectivas de regras e super- } \\
\text { visão de políticas. }\end{array}$ \\
\hline
\end{tabular}

Fonte: Adaptação feita pelo autor do Quadro Seven major themes about the algorithm (RAINIE; ANDERSON, 2017, n.p.)

Expressando as inúmeras possibilidades de classificação das implicações algorítmicas pelas Ciências Humanas e Sociais, o trabalho de Martin Lodge e Andrea Mennicken (2017, p.2, tradução nossa) buscou, além das "questões de regulação da regulação algorítmica," trazer a dimensão das questões éticas para o terreno das políticas públicas e perguntar qual conhecimento deve ser priorizado para tratar dos efeitos potenciais da regulação realizada pelos algoritmos? O quadro a seguir, foi baseado no trabalho apresentado por Lodge e Mennicken (2017) e explora pontos positivos e negativos das ações realizadas e controladas pelos sistemas algoritmos.

Quadro 3 - Efeitos potenciais da regulação realizada pelos algoritmos

\begin{tabular}{|l|l|}
\hline Aleatoriedade artificial aumentada & Maior supervisão \\
+ torna os jogos e a corrupção menos viáveis, \\
$\begin{array}{l}\text { pois os reguladores podem processar vastos } \\
\text { fluxos de informações em vez de confiar em } \\
\text { indicadores-chave. }\end{array}$ & $\begin{array}{l}+ \text { torna as avaliações baseadas em risco mais } \\
\text { prováveis, pois vastos fluxos de informações } \\
\text { permitem análises mais refinadas e supervisão } \\
\text { sob medida. }\end{array}$ \\
$\begin{array}{l}\text { - Informações complexas e vastas podem reduzir } \\
\text { a possibilidade de detectar informações essen- } \\
\text { ciais / a não transparência dos algoritmos signi- } \\
\text { fica falta de compreensão dos padrões. }\end{array}$ & $\begin{array}{l}\text { - Aumenta substancialmente os poderes de intru- } \\
\text { são e vigilância. }\end{array}$ \\
\hline $\begin{array}{l}\text { Maior rivalidade } \\
+ \text { aumenta a possibilidade de classificação e } \\
\text { benchmarking. }\end{array}$ & $\begin{array}{l}\text { Maior mutualidade } \\
+ \text { aprimora informações para um envolvimento } \\
\text { informado. }\end{array}$ \\
$\begin{array}{l}\text { - Aumenta a vulnerabilidade a jogos e corrupção } \\
\text { por ataques de bots e malware. }\end{array}$ & $\begin{array}{l}\text { - Aumenta o domínio do “analista de dados" em } \\
\text { relação a outros tipos de conhecimento profissio- } \\
\text { nal / conversas tendenciosas. }\end{array}$ \\
\hline
\end{tabular}

Fonte: Adaptação feita pelo autor do Quadro1 do texto The importance of regulation of and by algorithm (LODGE; MENNICKEN, 2017, p.5. 
Sem dúvida, existem outras possibilidades de classificação dos problemas que envolvem ou justificam uma regulação e a governança de sistemas algorítmicos. Classificar implica em escolher o que incluir e o que excluir de cada divisão. A prática discursiva sobre a regulação algorítmica envolve uma série de enunciados que se interligam e se reforçam, mas existem aqueles que se contrapõe e se excluem. Esses discursos guardam ideologias. Karen Yeung (2017) escreveu que o "eu neoliberal", aqui interpretado como uma construção ideológica, seria o maior consumidor de serviços digitais, o foco da governança por sistemas algorítmicos. Todavia, Julio Cesar Lemes de Castro (2018, p.169) alerta que "em que pese seu elo com o projeto neoliberal, não é possível asseverar que a governança algorítmica seja uma prerrogativa dele".

\section{Governança algorítmica e totalitarismos}

O pesquisador Henrique Félix de Souza Machado publicou, em 2018, a primeira compilação realizada no Brasil sobre regulação e governança algorítmica. Ao utilizar o Google Scholar acabou analisando 45 textos que apareciam na busca e se relacionavam com o tema. Constatou uma presença maior nas análises realizadas da tradição foucaultiana (WILLIAMSON, 2013; ROUVROY, 2014; MCQUILLAN, 2015), da teoria do Ator-Rede (INTRONA, 2013; MUSIANI 2013; CAMPBELL-VERDUYN et al., 2016) e da perspectiva institucionalista e regulatória (WILLIAMSON, 2014; YEUNG, 2016), além do que reuniu como visões da "economia, negócios e produção colaborativa" e auto governança (O’REILLY, 2013; PITT et al., 2014), entre outras. O recorte e enquadramento são bastante discutíveis, mas Machado (2018) apresentou uma grande contribuição ao debate acadêmico. A perspectiva marxista estava ausente ou sob forte nebulosidade no debate e no discurso científico sobre os sistemas algorítmicos. Em 2019, Nick Dyer-Witheford, Atle Mikkola Kjøsen and James Steinhoff lançam o livro Inhuman Power: Artificial Intelligence and the Future of Capitalism em que trazem o debate marxista para tentar compreender se o que os sistemas algorítmicos realizam é ou não trabalho.

O discurso acadêmico não tem vinculado sistemas algorítmicos com a teoria do totalitarismo, mas com a teoria econômica sobre o capitalismo, em especial, com sua vertente neoliberal. A concorrência e as possibilidades de vazio regulatório têm levado as corporações a agirem no sentido de capturar o máximo de dados possíveis, o que fundamenta uma coleta cada vez mais ubíqua e intrusa no cotidiano, público e íntimo, de cada pessoa, consumando uma situação de vigilância contínua (ZUBOFF, 2019). Além disso, um conjunto de contraposições que envolvem a segmentação e as profundas assimetrias aparecem no discurso já no início da segunda década do 
século XXI (MAGNET, 2011), mas ganham corpo no último quarto da década, envolvendo a questão da segregação da pobreza, da discriminação de classe, de raça e gênero, entre outras (EUBANKS, 2018; NOBLE, 2018, BENJAMIN, 2019).

Olivia J Erdélyi e Judy Goldsmith (2018) trazem uma dimensão discursiva polêmica, mas promissora, ao recomendarem cautela em regulações puramente nacionais dos sistemas de Inteligência Artificial. As pesquisadoras defendem a criação de uma estrutura de cooperação regulatória internacional na área de IA que seja viável e permita afastar políticas fragmentadas de regulação da AI que gerem tensões internacionais. Erdélyi e Goldsmith (2018) consideram que a regulamentação da IA possui externalidades que transcendem as fronteiras nacionais. Apesar de considerarem que normas legais têm origens sociais, as considerações éticas terão um papel central na formulação do corpo legal sobre a AI. O uso de algoritmos de inteligência artificial em armamentos e com fins bélicos deveria ser internacionalmente proibido. Ainda como exemplo, existem ameaças de grande perigo com a utilização antiética da IA, como nos aplicativos de reconhecimento de padrões (WANG, KOSINSKI, 2018).

Outra constatação importante no plano do discurso é que há um consenso em torno do tema ética. Pode aparecer no discurso empresarial o risco de a regulação precoce matar o avanço técnico-científico. Isso gera uma contraposição entre regulação e progresso tecnológico. O desenvolvimento dos sistemas algorítmicos, por exemplo, deveria ser regido pela ética e não por legislações, afirmam diversos líderes empresariais. A ética é cultural e historicamente condicionada, e o debate relacionado aos algoritmos ainda não adquiriu relevância. As principais contraposições estão no quadro a seguir.

Quadro 4-Contraposições discursivas

\begin{tabular}{|l|l|}
\hline OPACIDADE & TRANSPARÊNCIA / ACCOUNTABILITY \\
\hline INESCRUTABILIDADE & EXPLICABILIDADE \\
\hline $\begin{array}{l}\text { AUTONOMIA OPERACIONAL } \\
\text { SISTÊMICA }\end{array}$ & RESPONSABILIZAÇÃO HUMANA \\
\hline VIÉS E DISCRIMINAÇÃO & JUSTIÇA E REPARAÇÃO \\
\hline PROFILING E RANKING & AUDITORIA \\
\hline EFICIÊNCIA E EFICÁCIA & $\begin{array}{l}\text { DENÚNCIA DO TOTALITARISMO } \\
\text { NEOLIBERAL }\end{array}$ \\
\hline $\begin{array}{l}\text { SEGREGAÇÃO E EXCLUSÃO DE } \\
\text { SEGMENTOS DA SOCIEDADE }\end{array}$ & $\begin{array}{l}\text { TECNOPOLÍTICAS DE INCLUSÃO E } \\
\text { DEFESA DA DIGNIDADE SOCIAL }\end{array}$ \\
\hline
\end{tabular}

Fonte: autor. 


\section{Considerações finais: os regimes de verdade diante dos algoritmos}

David Beer (2017) chama a atenção para o pensar não apenas no impacto e nas consequências do código, mas também nas maneiras poderosas pelas quais as noções e as ideias sobre o algoritmo circulam pelo mundo social. Quando se analisa o discurso, o objetivo principal está nos efeitos de consciência e de percepção que acabam se tornando efeitos sociais tão concretos quanto qualquer força material. A filósofa Antoinette Rouvroy (2014) não desconhece tal processo de atualização do virtual, mas apresenta a hipótese de que existe uma crise de representação geral, uma impossibilidade de o sinal substituir a parte da realidade que quer representar (ROUVROY; STIEGLER, 2016). A partir da perspectiva foucaultiana de regimes de verdade, Rouvroy (2014) questiona se a verdade é possível diante dessa crise e da ideologia de objetividade trazida pela gestão algorítmica do Big Data.

O regime de verdade, no sentido apresentado por Foucault, que não expressa o que seria a verdade, mas as formas de validação do que pode ser considerado verdadeiro, indica que os sistemas algorítmicos, em suas roupagens de Big Data, Inteligência Artificial, Machine Learning, "vieram para ficar", como dita hoje o discurso nos corredores de vários departamentos da Academia. Esta pesquisa demonstra que a Academia possui uma diversidade de direções e de visões que aparecem claramente nos discursos consolidados em artigos e ensaios, seja considerando que os sistemas algorítmicos funcionam como um modo de controle, engendrando processos que nos identificam, modulam e regulam nossas vidas (CHENEY-LIPPOLD, 2011) ou que abalará as Ciências Sociais uma vez que "have lost their monopoly on making sense of empirical data, as big-data analysis replaces the highly skilled survey specialists of the past ". ${ }^{11}$ (MAYER-SCHÖNBERGER; CUKIER, 2013, 30).

Danah Boyd e Kate Crawford (2012) ao caracterizarem o Big Data como um fenômeno cultural, tecnológico e acadêmico que maximizando o poder computacional e a precisão algorítmica conduz a uma crença generalizada de que grandes conjuntos de dados oferecem uma forma mais alta de inteligência e conhecimento, fazem uma denúncia do mito da objetividade algorítmica que também é realizada de modo contundente pelo pesquisador Tarleton Gillespie (2014). O Google não precisa conhecer nenhuma língua para traduzi-la. Detecta padrões e utiliza a matemática sem recorrer a análise semântica ou causal. Os algoritmos de aprendizagem de máquina estariam alterando o modo de pensar e fazer a ciência. Assim, a impressão é de que a correlação seria capaz de substituir a causalidade. Em, 2006, Chris Anderson (2008) sintetizou o espírito de muitos pesquisadores ao

\footnotetext{
11 "eles perderam o monopólio de entender os dados sociais empíricos, pois a análise de big data substitui os especialistas em pesquisa altamente qualificados do passado". (MAYER-SCHÖNBERGER; CUKIER, 2013, p.30, tradução nossa).
} 
afirmar que diante de dados massivos, a hipótese, o modelo e as teorias estariam se tornando obsoletas.

A expansão dos sistemas algoritmicos, corporificados nas expressões Big Data, Machine Learning e Inteligência Artificial, com a finalidade de modernização, de fazer mais com menos, tornar a gestão mais eficaz e eficiente se torna uma verdade discursiva, mais recentemente contestada por pesquisas que mostram que tal discurso serve à segregação e à redução dos benefícios de segmentos mais pauperizados e fragilizados da sociedade.

\section{Discourse on REgULATION AND ALGORITHMIC GOVERNANCE}

ABSTRACT: The text presents the discursive practices of the main researches on the regulation and governance of algorithms. It exposes the fundamental elements which articulate the discourses around algorithmic systems and their expressions such as Big Data, machine learning and Artificial Intelligence. The main statements are identified and the controversies on regulation and the discursive contrasts about the risks and benefits of the generalized implementation of algorithmic systems are exposed in this text.

KEYWORDS: Algorithmic systems. Algorithmic governance. Algorithmic regulation. Algorithms. Artificial intelligence.

\section{DiscuRSOS SOBRE REGULACIÓN Y GOBERNANZA ALGORÍTMICA}

RESUMEN: El texto presenta las prácticas discursivas de las principales investigaciones sobre la regulación y el gobierno de los algoritmos. Discute los elementos fundamentales que articulan los discursos en torno a los sistemas algoritmicos y sus expresiones como: Big Data, machine learning e inteligencia artificial. Identifica las principales declaraciones y expone las controversias sobre la regulación y los contrastes discursivos sobre los riesgos y beneficios de la implementación generalizada de sistemas algoritmicos.

PALABRAS CLAVE: Sistemas algoritmicos. Gobierno algoritmico. Regulación algoritmica. Algoritmos. Inteligencia artificial. 


\section{REFERÊNCIAS}

ANDERSON, Chris. The end of theory: The data deluge makes the scientific method obsolete. Wired Magazine, v. 16, n. 7, 2008.

ANEESH, Aneesh. Global labor: Algocratic modes of organization. Sociological Theory, v. 27, n. 4, p.347-370, 2009.

BALDWIN, Robert; SCOTT, Colin; HOOD, Christopher. A reader on regulation. Oxford University Press, 1998.

BEER, David. The social power of algorithms. Information, Communication \& Society, 20:1, 1-13, 2017.

BEER, David. Power through the algorithm? Participatory web cultures and the technological unconscious. New Media \& Society, v. 11, n. 6, p. 985-1002, 2009.

BENJAMIN, Ruha. Race after technology: Abolitionist tools for the new jim code. John Wiley \& Sons, 2019.

BLACK, J. Learning from Regulatory Disasters. LSE Law, Society and Economy Working Papers, v. 24, 2014.

BOBBIO, Norberto. A democracia e o poder invisível. In: BOBBIO, Norberto. O futuro da democracia: uma defesa das regras do jogo. Rio de Janeiro: Paz e Terra, 1997.

BOYD, Danah; CRAWFORD, Kate. Critical questions for big data: Provocations for a cultural, technological, and scholarly phenomenon. Information, Communication \& Society, v. 15, n. 5, p.662-679, 2012.

CAMPBELl-VERDUYN, M.; GOGUEN, M.; PORTER, T. Big Data and algorithmic governance: the case of financial practices. New Political Economy, v. 22, n. 2, p.219-236, ago. 2016.

CASTRO, Julio Cesar Lemes de. Redes sociais como modelo de governança algorítmica (Social Networks as a Model of Algorithmic Governance). Matrizes, São Paulo, v. 12, n. 2, p.165-191, 2018.

CHENEY-LIPPOLD, John. A new algorithmic identity: Soft biopolitics and the modulation of control. Theory, Culture \& Society, v. 28, n. 6, p.164-181, 2011.

CITRON, Danielle Keats; PASQUALE, Frank. The scored society: Due process for automated predictions. Wash. L. Rev., v. 89, p.1, 2014.

COELHO, Lilian. Quais oportunidades a LGPD traz para os advogados? Blog da Aurum. Publicado em 20 de setembro de 2019. Disponível em: https://www.aurum.com.br/blog/ $\operatorname{lgpd} /$. Acesso em: 15 jun. 2020. 
DANAHER, John; HOGAN, Michael J.; NOONE, Chris; KENNEDY Rónán; BEHAN, Anthony; DE PAOR Aisling; FELZMANN, Heike; HAKLAY, Muki; KHOO, Su-Ming; MORISON, John; MURPHY, Maria Helen; O'BROLCHAIN, Niall; SCHAFER; Burkhard; SHANKAR, Kalpana. Algorithmic governance: Developing a research agenda through the power of collective intelligence. Big Data \& Society, v. 4, n. 2, 2017. Disponível em: https:// doi.org/10.1177/2053951717726554. Acesso em: 14 jun. 2020

DARDOT, Pierre; LAVAL, Christian. A nova razão do mundo. São Paulo: Boitempo Editorial, 2017.

DIAKOPOULOS, N. Algorithmic-Accountability: the investigation of Black Boxes. Tow Center for Digital Journalism, 2014.

DIAKOPOULOS, Nicholas. Accountability in algorithmic decision making. Communications of the ACM, v. 59, n. 2, p.56-62, 2016.

DIAKOPOULOS, Nicholas. Algorithmic accountability: Journalistic investigation of computational power structures. Digital journalism, v. 3, n. 3, p.398-415, 2015.

DOMINGOS, Pedro. The master algorithm: How the quest for the ultimate learning machine will remake our world. Basic Books, 2015.

DONEDA, Danilo; ALMEIDA, Virgilio AF. What is algorithm governance? IEEE Internet Computing, v. 20, n. 4, p.60-63, 2016.

DYER-WITHEFORD, Nick; KJØSEN, Atle Mikkola; STEINHOFF, James. Inhuman Power. Artificial Intelligence and the Future of Capitalism. Pluto Press, 2019.

ERDÉLYI, Olivia J.; GOLDSMITH, Judy. Regulating artificial intelligence: Proposal for a global solution. Proceedings of the 2018 AAAI/ACM. Conference on AI, Ethics, and Society. 2018.

EUBANKS, Virginia. Automating inequality: How high-tech tools profile, police, and punish the poor. St. Martin's Press, 2018.

GILLESPIE, Tarleton. The relevance of algorithms. In: GILLESPIE, Tarleton; BOCZKOWSKI, Pablo J.; FOOT, Kirsten A. Media technologies: Essays on communication, materiality, and society. MIT Press, 2014.

GOLDMAN, Eric. Search engine bias and the demise of search engine utopianism. Yale Journal of Law \& Technology, 8, p.188-200, 2006.

GOODMAN, Bryce; FLAXMAN, Seth. European Union regulations on algorithmic decision-making and a "right to explanation". AI Magazine, v. 38, n. 3, p.50-57, 2017. 
INTRONA, Lucas D. Algorithms, performativity and governability. Governing Algorithms: A Conference on Computation, Automation, and Control, New York University, May. 2013. p.16-17.

INTRONA, Lucas D.; NISSENBAUM, Helen. Shaping the Web: Why the politics of search engines matters. The information society, v. 16, n. 3, p.169-185, 2000.

LODGE, Martin; MENNICKEN, Andrea. The importance of regulation of and by algorithm. Algorithmic Regulation, p.2, 2017.

MACHADO, H. F. de S. Algoritmos, regulação e governança: uma revisão de literatura. Revista de Direito Setorial e Regulatório, Brasília, v. 4, n. 1, p.39-62, maio 2018.

MAGNET, Shoshana. When biometrics fail: Gender, race, and the technology of identity. Duke University Press, 2011.

MARSDEN, Christopher T. Internet co-regulation and constitutionalism: Towards European judicial review. International Review of Law, Computers \& Technology, v. 26, n. 2-3, p. 211-228, 2012.

MAYER-SCHÖNBERGER, Viktor; CUKIER, Kenneth. Big Data: A revolution that will transform how we live, work, and think. Houghton Mifflin Harcourt, 2013.

MCQUILLAN, D. Algorithmic States of Exception. European Journal of Cultural Studies, v. 18, n. 4-5, p.564-576, jun. 2015.

MUSIANI, F. Governance by algorithms. Internet Policy Review, v. 2, n. 3, p.1-8, ago. 2013.

NOBLE, Safiya Umoja. Algorithms of oppression: How search engines reinforce racism. NYU Press, 2018.

O'REILLY, Tim. Open data and algorithmic regulation. In: GOLDSTEIN, Brett; DYSON, Lauren. Beyond transparency: Open data and the future of civic innovation, p.289-300, 2013.

PASQUALE, Frank. A esfera pública automatizada. LÍBERO, v. 20 - no 39 jan./ago. p.1635, 2017. Disponível em: http://seer.casperlibero.edu.br/index.php/libero/article/view/866. Acesso em: 15 jun. 2020.

PASQUALE, Frank. The black box society. Harvard University Press, 2015.

PITT, J.; BUSQUETS Dídac; DIACONESCU, Ada; NOWAK Andrzej; RYCHWALSKA Agnieszka; ROSZCZYNSKA-KURASINSKA, Magda. Algorithmic self-governance and the design of socio-technical systems. CEUR Workshop Proceedings, v. 1283, p.262-273, 2014. Disponível em: http://ceur-ws.org/Vol-1283/paper_33.pdf. Acesso em: 3 dez. 2017. 
RAINIE, Lee; ANDERSON, Janna. Code-Dependent: Pros and Cons of the Algorithm Age. Pew Research Center. February 8, 2017. Disponível em: https://www.pewresearch.org/ internet/2017/02/08/code-dependent-pros-and-cons-of-the-algorithm-age/. Acesso em: 15 jun. 2020.

ROUVROY, A. Data Without (Any)Body? Algorithmic governmentality as hyperdisadjointment and the role of Law as technical organ. General Organology Conference, University of Kent, Canterbury, Reino Unido, nov. 2014.

ROUVROY, Antoinette; STIEGLER, Bernard. The digital regime of truth: from the algorithmic governmentality to a new rule of law. La Deleuziana: Online Journal of Philosophy, v. 3, p.6-29, 2016. Disponível em: http://www.ladeleuziana.org/wp-content/ uploads/2016/12/Rouvroy-Stiegler_eng.pdf. Acesso em 17 jun. 2020.

SANDVIG, Christian; HAMILTON, Kevin; KARAHALIOS; Karrie; LANGBORT, Cedric. An algorithm audit. Data and discrimination: collected essays. New York, NY: New America, Open Technology Institute, p.6-10, 2014.

SILVEIRA, Sérgio Amadeu. A noção de modulação e os sistemas algorítmicos. In: SOUZA Joyce; AVELINO, Rodolfo; SILVEIRA Sérgio Amadeu da. (Org.). A sociedade de controle. Manipulação e modulação nas redes digitais. 1ed. São Paulo: Hedra, 2019.

THALER, Richard H.; SUNSTEIN, Cass R. Nudge: Improving decisions about health, wealth, and happiness. Penguin, 2009.

WANG, Yilun; KOSINSKI, Michal. Deep neural networks are more accurate than humans at detecting sexual orientation from facial images. Journal of personality and social psychology, v. 114, n. 2, p.246, 2018.

WHITE HOUSE OFFICE. White House summit on artificial intelligence for american industry. MAY 10, 2018.

WILLIAMSON, B. Knowing public services: Cross-sector intermediaries and algorithmic governance in public sector reform. Public Policy and Administration, v. 29, n. 4, p.292-312, may 2014.

WILLIAMSON, B. Decoding identity: Reprogramming pedagogic identities through algorithmic governance. Paper presented at British Educational Research Association conference, University of Sussex, Brighton, Reino Unido, 3 set. 2013.

YEUNG, K. Algorithmic Regulation: A Critical Interrogation. Regulation \& Governance. July, 2017. Disponível em: https://doi.org/10.1111/rego.12158. Acesso em: 16 jun. 2020.

YEUNG, K. Algorithmic regulation and intelligent enforcement. Centre for Analysis of Risk and Regulation Discussion Paper Series, n. 84, p.50-61, out. 2016. 
ZARSKY, Tal. The trouble with algorithmic decisions: An analytic road map to examine efficiency and fairness in automated and opaque decision making. Science, Technology $\&$ Human Values, v. 41, n. 1, p.118-132, 2016.

ZARSKY, Tal Z. Transparent predictions. Illinois Law Review, p.1503-1569, 2013. Disponível em: https://illinoislawreview.org/print/volume-2013-issue-4/transparentpredictions/. Acesso em: 16 jun. 2020.

ZUBOFF, Shoshana. The age of surveillance capitalism: The fight for a human future at the new frontier of power. Profile Books, 2019.

Recebido em 01/04/2020.

Aprovado em 13/04/2020. 
